\title{
Investor sentiment and stock returns: Some international evidence
}

\author{
Maik Schmeling \\ Discussion Paper No. 407 \\ November 2008 \\ ISSN 0949-9962
}

\begin{abstract}
:
We examine whether consumer confidence - as a proxy for individual investor sentiment affects expected stock returns internationally in 18 industrialized countries. In line with recent evidence for the U.S., we find that sentiment negatively forecasts aggregate stock market returns on average across countries. When sentiment is high, future stock returns tend to be lower and vice versa. This relation also holds for returns of value stocks, growth stocks, small stocks, and for different forecasting horizons. Finally, we employ a cross-sectional perspective and provide evidence that the impact of sentiment on stock returns is higher for countries which have less market integrity and which are culturally more prone to herd-like behavior and overreaction.
\end{abstract}

Keywords: consumer confidence; growth stocks; investor sentiment; noise trader; predictive regressions; value stocks

JEL: G12, G14, G15

* Maik Schmeling, Department of Economics, Institute of Money and International Finance. Leibniz Universität Hannover, Königsworther Platz 1, 30167 Hannover. Phone: (+49) 511 762-8213, e-mail: schmeling@gif.uni-hannover.de 


\section{Investor sentiment and stock returns: Some international evidence}

\section{Introduction}

Recent academic literature has seen a rise of studies investigating the effect of individual investor sentiment on stock returns. Several papers document a strong link between the two variables both in the time-series and cross-sectionally. These papers estimate predictive regressions of the form

$$
r_{t+1}=\alpha+\beta \cdot \text { sentiment }_{t}+\eta_{t}
$$

where $r_{t+1}$ is the return of the aggregate stock market or a (zero-cost) portfolio at time $t+1$ and sentiment $_{t}$ is a proxy for (lagged) investor sentiment. A common finding for the US stock market is a statistically and economically significant negative coefficient estimate for $\beta$. Therefore, periods of higher investor optimism tend to be followed by significantly lower returns for the aggregate market (e.g. Brown and Cliff, 2005) and even more pronouncedly for firms that are hard to price and thus difficult to arbitrage (e.g. Baker and Wurgler, 2006, Lemmon and Portniaguina, 2006).

Earlier evidence on the effects of sentiment almost exclusively focuses on crosssectional results for the U.S. stock market, e.g. for value versus growth stocks or small versus large stocks. Extending this earlier evidence, we analyze the effects of investor sentiment on international aggregate stock markets. An analysis of this sort seems interesting for several reasons.

First, stock markets at the aggregate country level are clearly both hard to value and hard to arbitrage. This follows from the fact that macro data is notoriously noisy and since it is difficult to hedge away idiosyncratic shocks at the country level. Therefore, it seems 
reasonable that sentiment shocks affect stock markets on aggregate and not just different subgroups of stocks. ${ }^{1}$

Second, taking an international perspective allows us to test new hypotheses relating to the effects of sentiment on returns. In this paper, we will test whether sentiment effects are especially pronounced in countries with low institutional development (e.g. La Porta et al., 1998) or in countries which are especially prone to herd-like behavior and overreaction (Chui, Titman and Wei, 2008).

Finally, using international stock return data provides a natural out-of-sample test for earlier U.S. findings (see e.g. Griffin, Ji, Martin (2003) or Ang et al. (2008) on the importance of testing market anomalies out-of-sample) and pooling data across countries increases the power of tests which yields more reliable estimates (see e.g. Ang and Bekaert (2007) for a discussion of this issue).

Therefore, we investigate whether consumer confidence - as a proxy for individual investor sentiment - affects stock returns along the lines of (1) in 18 countries around the globe. We find, first, that there is a significant impact of investor sentiment on aggregate stock returns across countries on average. This effect remains significant even after controlling for other standard risk factors and expected business conditions. Secondly, in cross-sectional regressions we provide some first evidence that the impact of sentiment on stock returns is stronger in countries that have less market integrity and in countries that are culturally more prone to herd-like behavior as predicted by Chui, Titman and Wei (2008).

The rest of this paper is structured as follows. The next section selectively reviews the existing literature and derives testable hypotheses. Section 3 describes the data and provides some descriptive statistics. Section 4 provides estimates of predictive regressions of returns

\footnotetext{
${ }^{1}$ Shiller (2001, p.243) actually quotes Paul Samuelson with the following claim: "I [hypothesize] considerable macro inefficiency, in the sense of long waves in the time series of aggregate indexes of security prices below and above various definitions of fundamental values."
} 
on sentiment similar to equation (1). Finally, section 5 investigates cross-country results and section 6 concludes.

\section{Earlier Literature and Testable Hypotheses}

The general finding of a sentiment-return relation is at odds with standard finance theory which predicts that stock prices reflect the discounted value of expected cash-flows and that irrationalities among market participants are erased by arbitrageurs. Sentiment does not play any role in this classic framework. Instead, the behavioral approach suggests that waves of irrational sentiment, i.e. times of overly optimistic or pessimistic expectations, can persist and affect asset prices for significant periods of time. ${ }^{2}$

Baker and Wurgler (2006) point out that sentiment-based mispricing is based on both an uninformed demand shock and a limit to arbitrage. Regarding the first ingredient, uninformed demand shocks, Brown and Cliff (2005) argue that sentiment is most likely a very persistent effect so that demand shocks of uninformed noise traders may be correlated over time to give rise to strong and persistent mispricings. ${ }^{3}$ However, the second ingredient, limits of arbitrage, deter informed traders from eliminating this situation (cf. Black, 1986, or more formally, Shleifer and Vishny, 1997) since it is a priori unclear how long buying or selling pressure from overly optimistic or pessimistic noise traders will persist. However, every mispricing must eventually be corrected so that one should observe that high levels of investor optimism are on average followed by low returns and vice versa.

Earlier evidence (e.g. Brown and Cliff, 2006) do indeed show that there is a negative sentiment-return relation on the aggregate U.S. stock market level. We investigate this relation for an international set of markets which leads to our first hypothesis:

\footnotetext{
${ }^{2}$ See also DeLong, Shleifer, Summers and Waldmann (1990) who show theoretically that correlated sentiment of noise traders affects equilibrium stock returns.

${ }^{3}$ Barber, Odean, and Zhu (2008a) document that trading by individuals is highly correlated which is consistent with systematic noise trading that does not wash out in the aggregate.
} 


\section{Hypothesis 1:}

International investor sentiment predicts future aggregate market returns. The relation between sentiment and expected returns is significantly negative and robust to controlling for fundamental factors.

There is also ample evidence (Baker and Wurgler, 2006) that sentiment affects the cross-section of returns differently for different investment styles, e.g. value and growth stocks or small and large stocks. This result naturally follows from the fact that e.g. small or growth stocks are harder to arbitrage and harder to value than large stocks with a long and stable earnings history. Baker and Wurgler for example find that sentiment effects are stronger among stocks that can reasonably be assumed to fulfill at least one of these criteria, e.g. young, small, unprofitable, distressed, extreme growth or dividend-nonpaying firms. For the U.S., Kumar and Lee (2006) show that retail investors, which are commonly thought of being noise traders, tend to overweight value stocks relative to growth stocks and that shifts in the buy-sell imbalance of these retail investors are positively correlated with returns of value stocks. This clearly is a prime example of noise trader risk.

Barber, Odean and Zhu (2008) investigate returns of stocks that are heavily traded by U.S. individuals and provide direct evidence on the hypothesis that individuals are noise traders. They show that stocks heavily sold by individuals outperform stocks heavily bought by a hefty $13.5 \%$ the following year. They also document strong herding among individual investors so that the notion of correlated trading by irrational investors seems to be a likely cause for these return differentials. ${ }^{4}$ Hvidkjaer (2008) finds similar results over horizons of up to three years.

We therefore test for such cross-sectional effects in an international setting and state our second hypothesis as follows.

\footnotetext{
${ }^{4}$ It should be noted, however, that Kaniel, Saar, and Titman (2008) find that net buying and selling pressure of individuals positively predicts future returns over short horizons of up to one month. This result casts some doubt on other studies relating to individual investor trading and subsequent returns.
} 


\section{Hypothesis 2:}

The effect of sentiment on returns is stronger for stocks that are hard to value and/or hard to arbitrage, e.g. growth stocks, value stocks, and small stocks.

Recently, Chui, Titman and Wei (2008) propose that cultural differences might play a role for the relative strength of behavioral biases between countries. ${ }^{5}$ Specifically, they argue that individualism as measured by Hofstede (2001) drives certain behavioral biases that are assumed to generate momentum profits. The authors also argue that a lack of individualism, i.e. collectivism, might drive biases that generate other market inefficiencies than the momentum premium. Collectivistic countries have societies in which people are integrated into strong groups. This structure leads to an overweighing of consensus opinion, and thus to "herd-like overreaction" (see Chui, Titman and Wei, 2008, p.28). Herd-like overreaction, i.e. correlated actions of noise traders based on overly optimistic or pessimistic expectations, is precisely what is assumed to drive the sentiment-return relation in financial markets. Therefore, one may expect that stock markets in collectivistic countries are more heavily influenced by investor sentiment whereas stock markets in individualistic, in which people tend to put more weight on their own information and opinion, should be less affected by these behavioral biases.

Finally, there is an extensive literature investigating how market quality affects market outcome (La Porta, Lopez-de-Silanes, Shleifer, and Vishny, 1998). Therefore, we also check whether institutional quality of a country explains the cross-section of the sentiment-return relation. This results in our third hypothesis.

\section{Hypothesis 3:}

\footnotetext{
${ }^{5}$ Guiso, Sapienza and Zingales (2006) document that culture may significantly affect economic outcome although yet little attention has been paid to these factors in economics. However, there seems to be even less empirical evidence for the role of culture in finance than in economics.
} 
The impact of sentiment on returns is stronger for countries that have less well developed market institutions and for countries that are culturally more prone to investor overreaction.

\section{Data and Descriptive Statistics}

\subsection{General data considerations}

As previously noted, we are interested in measuring the effect of noise trader demand shocks on stock markets. Doing this in a consistent way is exacerbated by the fact that there is no consensus on what kind of proxies to employ when measuring individual sentiment for a single country. This problem naturally aggravates when attempting to find a proxy that is available for different countries.

However, given the recent detailed analysis of consumer confidence as a proxy for investor sentiment by Lemmon and Portniaguina (2006) it seems natural to use this metric for an international analysis. First of all, consumer confidence is available for several industrialized countries and, secondly, it is available for reasonable periods of time. Third, consumer confidence, albeit measured slightly different in various countries, seems to be the only consistent way to obtain a sentiment proxy that is largely comparable across countries and that is not calculated from trading data itself. ${ }^{6}$

Therefore, we use data on stock returns and consumer confidence for 18 industrialized countries to investigate the international sentiment-return relation. Our sample of countries is largely dictated by data availability but consumer confidence is available for several countries on horizons of up to 20 years. We include the U.S., Japan, Australia, New Zealand and 14 European countries (see Table 1 for a complete list of countries). These markets cover the lion's share of international stock market capitalization, cover the most liquid markets in the

\footnotetext{
${ }^{6}$ A short overview of alternative sentiment measures and further evidence on consumer confidence as a proxy for investor sentiment can be found in Appendix 1.
} 
world - namely the U.S., Europe and Japan - and thus provide a representative sample. Some details on international consumer confidence indices can be found in Appendix 2.

For each of the 18 countries we collect a monthly measure of consumer confidence, monthly returns for (a) the aggregate stock market, (b) a portfolio of value stocks and (c) a portfolio of growth stocks. ${ }^{7}$

\subsection{Descriptive statistics}

Stock market data come from Professor Kenneth French's web site and are employed because they are collected in a consistent manner across countries, are relatively free of survivorship bias (Fama and French, 1998) and were used in other studies before (Chui, Titman and Wei (2008) motivate their herding and collectivism result with this data).

Furthermore, for each country we collect data on consumer confidence. For all 14 European countries the data comes from the "Directorate Generale for Economic and Financial Affairs" (DG ECFIN) ${ }^{8}$ which, among other things, conducts research for the European Union. Confidence indices for the remaining countries are obtained from Datastream or EcoWin. There are several possible high-quality consumer confidence indices for the U.S. We employ the Michigan Survey (see e.g. Lemmon and Portniaguina, 2006). Finally, the consumer confidence index for Japan is available on a quarterly frequency only. We convert it to a monthly frequency by using the last available values for months without data as in Baker and Wurgler (2006).

Table 1 provides descriptive statistics for returns and consumer confidence indices. Column three shows the periods available for each country. We include the time from January 1985 to December 2005 wherever possible. Data limitations enforce somewhat shorter

\footnotetext{
${ }^{7}$ Stock market returns are from value-weighted portfolios in local currency. The value portfolio consists of the top three deciles of stocks sorted by B/M (book-to-market ratio) whereas the growth portfolio comprises the bottom $30 \%$ of stocks sorted by $\mathrm{B} / \mathrm{M}$.

${ }^{8}$ These consumer confidence indices have also been used by Jansen and Nahuis (2003). Data can be downloaded from: http://ec.europa.eu/economy_finance/indicators_en.htm.
} 
periods for several countries. However, we have a minimum of 120 monthly observations even for the most data-constrained country Austria.

As can be seen, value stocks have higher mean returns than growth stocks for most countries, a fact documented before in a voluminous literature on the value premium (Fama and French, 1998). The descriptive statistics for the consumer confidence indices show a high degree of serial correlation in the time-series. First order autocorrelations $\left(\rho_{-1}\right)$ are high and uniformly above $90 \%$. We will take special care of the serial correlation in our empirical analyses.

\subsection{Some preliminary tests}

Given these large autocorrelations it appears interesting to test whether our consumer confidence indices are unit-root non-stationary. We present panel unit-root tests in Table 2 that (a) test for a common unit root (Levin, Lin, and Chu, 2002) or (b) for individual unitroots in the 18 consumer confidence series. Results shown in Table 2 are comforting and suggest that we indeed deal with stationary, but highly persistent, time-series processes.

It is also reassuring, that correlation coefficients of the consumer confidence indices (not reported here for the sake of brevity) are not prohibitively strong, i.e. we are not using essentially one sentiment series for all countries. More precisely, cross-country correlation coefficients range from -0.47 to 0.88 . Consequently, there are several countries that show a large correlation (e.g. Austria and Germany), essentially no correlation (e.g. Australia and Switzerland) or a negative correlation (e.g. Sweden and Japan). These results hold for both levels and changes of consumer confidence series.

Finally, we take a look at Granger-Causality tests as a simple device to check for timeseries dependencies between our sentiment measures and stock returns. Results for simple bivariate (stock returns and consumer confidence) Granger-Causality tests and Block 
exogeneity tests are shown in Table $3 .{ }^{9}$ As can be inferred, there is two-way "causality" such that sentiment depends on previous returns and that returns depend on previous sentiment movements. ${ }^{10}$ How can these findings be interpreted? As Qiu and Welch (2005) point out, sentiment should be related to some variable, e.g. returns, macro variables, etc. This just follows from the fact that sentiment "should not fall like manna from heaven" (Qiu and Welch, 2005, p. 23). Rather investors are overly optimistic or pessimistic due to a series of good or bad news, returns, or macro developments. Therefore, the result that returns drive sentiment and that sentiment drives subsequent returns, seems very reasonable. Also, the causality running from sentiment to returns may be seen as a first confirmation of hypotheses 1 and 2.

\section{Predictive Regressions of Stock Returns on Consumer Confidence}

This section presents results on the sentiment-return relation. We will first introduce the econometric methodology in section 4.1, present results from panel regressions in section 4.2, discuss findings for individual countries in section 4.3, and relate our findings for international markets to earlier studies in section 4.4 .

\subsection{Methodology}

In order to test for sentiment effects on future returns, we estimate long-horizon return regressions of the form

$$
\frac{1}{\kappa} \sum_{\kappa=1}^{\mathrm{k}} \mathrm{r}_{\mathrm{t}+\mathrm{\kappa}}^{\mathrm{i}}=\delta_{0}^{\mathrm{i},(\mathrm{k})}+\delta_{1}^{\mathrm{i},(\mathrm{k})} \operatorname{sent}_{\mathrm{t}}^{\mathrm{i}}+\Psi_{\mathrm{t}}^{\mathrm{i},(\mathrm{k})} \gamma^{\mathrm{i},(\mathrm{k})}+\xi_{\mathrm{t}+1 \mapsto \mathrm{t}+\mathrm{k}}^{\mathrm{i},(\mathrm{k})}
$$

\footnotetext{
${ }^{9}$ Tests for block exogeneity are based on VARs that include several control variables, such as dividend yields, CPI inflation, industrial production, detrended short rates and term spreads. Estimation of the underlying VARs is done by pooling data of all countries in the sample. Each country has its own vector of constants while slope coefficients are restricted to be equal across countries.

${ }^{10}$ It should be noted, that Brown and Cliff (2004) also test for Granger-Causality between sentiment and returns. They find that causality runs from small and large stock returns to sentiment and that sentiment impacts returns of small but not of large stocks.
} 
with the average $k$-period return ${ }^{11}$ for country $i$ as dependent variable and several predictors on the right-hand side. These predictors include consumer confidence as a proxy for individual sentiment (sent) and additional macro variables which are collected in matrix $\Psi$. Specifically, we include annual CPI inflation, the annual percentage change in industrial production, the term spread, the dividend yield, and the detrended (6 months) short rate in $\Psi$ to net out effects of commonly employed risk factors on returns. ${ }^{12}$ As usual, we employ known up-to-week $t$ information to forecast mean excess returns beginning in month $t+1$ only. Furthermore, to facilitate comparisons of the sentiment-return relation between countries we standardize all right-hand side variables used in (2).

Based on the general predictive regression in (2), we will pursue two different approaches. First, we estimate panel fixed-effects regressions, so that all countries enter the regressions jointly. The cross-sectional fixed-effects specification allows individual countries to have different regression constants. We use panel regressions to increase the power of our tests and to investigate whether there is a significant sentiment-return relation on average across countries. This approach translates into

$$
\frac{1}{\kappa} \sum_{\mathrm{k}=1}^{\mathrm{k}} \mathrm{r}_{\mathrm{t}+\mathrm{\kappa}}^{\mathrm{i}}=\delta_{0}^{\mathrm{i},(\mathrm{k})}+\delta_{1}^{(\mathrm{k})} \operatorname{sent} \mathrm{t}_{\mathrm{t}}^{\mathrm{i}}+\Psi_{\mathrm{t}}^{(\mathrm{k})} \gamma^{\mathrm{i},(\mathrm{k})}+\xi_{\mathrm{t}+1 \mapsto \mathrm{t}+\mathrm{k}}^{\mathrm{i},(\mathrm{k})}
$$

so that there is a country-specific intercept. However, slope coefficients are restricted to be equal across countries.

Second, we estimate (2) separately for each of the 18 countries in our sample and test for a significant impact of sentiment on future returns across horizons. More specifically, we jointly estimate equation (2) for forecast horizons of 1, 6, 12, and 24 months in a system of regression equations and perform tests of the form $\delta_{1}^{\mathrm{i}, 1}=0, \delta_{1}^{\mathrm{i}, 6}=0, \delta_{1}^{\mathrm{i}, 12}=0, \delta_{1}^{\mathrm{i}, 24}=0$, i.e. we

\footnotetext{
${ }^{11}$ As in Hong et al. (2007) we use raw returns since reliable data on risk-free rates is hard to obtain outside the U.S.

${ }^{12}$ Baker and Wurgler (2006) and Lemmon and Portniaguina (2006) also net out macro risk factors from their sentiment proxy to obtain an explanatory variable that is unrelated to fundamental risk factors.
} 
test whether there is a jointly significant impact at the 1, 6, 12, and 24 months horizon. This joint test of predictability at different forecast horizons has been employed earlier in the literature (see e.g. Mark (1995) for an early and Ang and Bekaert (2007) for a more recent application) and is a more reasonable device to test for predictability than just testing at individual forecast horizons. This follows naturally from the fact that results are correlated across horizons.

Finally, we have to deal with some technical problems arising in predictive regressions of the form employed here. These problems stem from persistent predictive variables (Stambaugh, 1999) and from employing overlapping observations. We use a moving block-bootstrap simulation procedure to overcome these problems. Details on the simulation can be found in Appendix 3.

\subsection{Results for panel regressions}

We start with the results for fixed-effects panel regressions which are shown in $\underline{\text { Table }}$ 4. The three Panels of this table provide results for the aggregate market (Panel A), value stocks (Panel B), and growth stocks (Panel C). The coefficients reported directly show the impact of a two standard deviation shock (i.e. a "large shock") of sentiment on returns. Looking at Panel A first, it can be seen that investor sentiment has a significantly negative impact on future stock returns at all forecast horizons (1 to 24 months) although significance is limited to the $6 \%$ level at the longest horizon. Overall, the result of a significantly negative relation between sentiment and returns is in line with theoretical considerations of the impact of noise traders and earlier empirical findings for the U.S. and our first hypothesis.

Furthermore, it is interesting to note that the impact of sentiment on average future returns declines with the forecast horizon. This finding has both a statistical and an economic implication. First, it is often argued that long-horizon regressions with nearly integrated regressors spuriously generate significant results at increasing horizons (cf. Hong et al. 
(2007), p. 17 for a discussion). If there was a bias in our results not eliminated by the bootstrapping procedure that mechanically generated significant results over longer horizons, one would expect to see exactly such a result. Yet, this is not the case here. Second, in economic terms, the diminishing marginal impact suggests that noise trading effects do indeed wash out over longer time periods, i.e. there are limits to arbitrage in the short to medium run but these limits to arbitrage become weaker at longer horizons. This result seems reasonable from a theoretical viewpoint since the opposite finding would mean that noise trader demand shocks move markets permanently away from equilibrium.

Results are also significant in economic terms. A two standard deviation rise in the sentiment proxy depresses stock returns by $0.40 \%$ on average over the next month and, although the average monthly effect declines with the forecast horizon, has a negative impact of almost $5 \%$ over the following two years $(-0.20 \times 24$ months $)$. Looking at the adjusted $\mathrm{R}^{2} \mathrm{~s}$ and incremental adjusted $R^{2}$ s (i.e. the rise in adj. $R^{2} s$ when additionally including the sentiment factor in the predictive regression) reveals that sentiment adds quite some explanatory power relative to the other predictor variables, especially at short and intermediate horizons. For example, at the one month horizon, sentiment accounts for all of the explained variance (although the $\mathrm{R}^{2}$ is small which is typical for stock return forecasts).

Panels B and C of Table 4 show results for value and growth stocks. Findings are qualitatively similar to those for aggregate market returns, but the effects for value stocks are much more pronounced than for aggregate markets or growth stocks. For example, a one standard deviation shock in consumer confidence leads to a decrease in average expected returns of more than $0.5 \%$ p.m. over a six months horizon for value stocks (Table 4, Panel B) whereas aggregate market returns and growth stocks decline by only $0.3 \%$ p.m. on average. Therefore, hypothesis 2 receives some support in general but the results suggest that value stocks are more heavily influenced than growth stocks. 


\subsection{Results for individual countries}

Results for the predictive power of sentiment in individual countries are presented in Table 5, Panels A to C. The Table reports the average predictive coefficient of sentiment over forecast horizons of $1,6,12$, and 24 months and the p-value of a test that all forecast coefficients are zero.

As can be seen from Panel A, which provides results for aggregate market returns, there is quite some heterogeneity across countries. All in all, a significant sentiment-return relation is found for 9 countries on a 5\%-level of significance and for 11 countries on a $10 \%$ level only, which roughly equals $50 \%$ of countries in our sample. Therefore, sentiment effects on returns seem to be country-specific and hypothesis 1 is not supported for all countries in our sample. A similar finding holds for value and growth stocks - shown in Panels B and C which also document that there is heterogeneity across countries. However, corroborating the evidence above, the sentiment-return relation seems somewhat stronger for value stocks than for growth stocks (and the aggregate market) as there are more countries with a significantly negative impact of sentiment on future returns. ${ }^{13}$ Therefore, hypothesis 2 cannot be accepted in general but receives more support for value stocks and less support for growth stocks.

Looking at the effects across countries, there is an especially strong relation between sentiment and future returns in countries such as Japan, Italy, or Germany, while there is little to no evidence for such effects in countries like Australia, New Zealand, or the U.K. Also, the U.S. cannot be seen as a country that is particularly prone to sentiment effects. The average coefficient estimate across forecasting horizons is only -0.12 and thus well below average. Several other European countries (e.g. Italy, Germany, or France) as well as Japan show a much larger impact of sentiment on returns.

\footnotetext{
${ }^{13}$ There are $10(13)$ countries with a significant impact of sentiment on returns on a $5 \%(10 \%)$ level for value stocks and $8(11)$ countries with a significant impact on a $5 \%(10 \%)$ level for growth stocks.
} 
In a nutshell, evidence does not seem to be obviously related to geographical locations or the size of a country.

\subsection{Relation of our results to earlier studies}

This section relates our findings to effects documented in the earlier literature. However, while most of earlier studies deal exclusively with the U.S., some caution is warranted when comparing these results with our findings for international stock markets.

First, the paper by Brown and Cliff (2005) provides some direct benchmark, since they provide results for the aggregate stock market, value, and growth stocks separately. Looking at their results (Table 6, p. 422) for the aggregate market, they find that a two standard deviation movement in their sentiment measure leads to declining returns of about $1.76 \%$ for a 6 -months horizon, and $-5.8 \%$ for a 12 -months horizon. These numbers are similar to our panel estimates of about $-1.86 \%$ and $-5.4 \%$ for the same horizons and thus seem to be of reasonable size. ${ }^{14}$

A different picture naturally emerges for the sentiment effects of value and growth stocks since the earlier literature does not provide consistent findings on this topic. While Brown and Cliff (2005) find a stronger effect for growth than for value stocks, Baker and Wurgler (2006) show for their sentiment proxy, that sentiment effects are of similar size for both value and growth stocks (see e.g. their Figure 2, p. 1663). Finally, Lemmon and Portnaguina (2006, p. 1526) provide evidence that sentiment effects are significant for value but not for growth stocks. Our general findings from panel regressions are consistent with Baker and Wurgler in that we find a significant effect of sentiment on both groups of stocks and in line with Lemmon and Portniaguina since the effect is stronger for value stocks. However, our results differ in that there is a significant effect for growth stocks (different

\footnotetext{
${ }^{14}$ These numbers are for panel estimates and thus represent average effects across countries. Our estimates for the U.S. (Table 5) show somewhat lower estimates of about $-1.2 \%$ (6-months horizon) and $-2.4 \%$ (12-months horizon).
} 
from Lemmon and Portniaguina) and that the effects are economically much stronger for value stocks (different from Baker and Wurgler). Therefore, our international results do not exclusively support any of the earlier findings in the U.S. literature. The differing results are likely to stem from the fact that we are using consumer confidence as a proxy for investor sentiment whereas earlier papers use a variety of different sentiment proxies and sample periods. Therefore, since U.S. results themselves are not fully consistent with each other, it is obvious that our results cannot be consistent with each of the earlier findings. Apart from this, our results suggest that earlier U.S. findings on cross-sectional effects cannot easily be generalized to international markets.

However, our individual results for the U.S. (from Table 5), are consistent with findings from Lemmon and Portniaguina who also find that sentiment significantly affects value stocks but not growth stocks. This result seems comforting since we are using the same proxy for investor sentiment as these authors.

Finally, we note that our results for individual countries are in line with the scant earlier evidence for different countries. As in our results, there is a significant effect for the U.S. (as discussed above), Jackson (2004) finds no significant evidence for noise trader induced return reversals in Australia while Schmeling (2006) finds evidence for a significant impact of individual sentiment on aggregate market returns in Germany.

\subsection{Robustness tests}

Many earlier papers on sentiment and stock returns have also looked at sentiment and the returns to small and large stocks. While we are primarily interested in the effect of sentiment on international aggregate stock markets, we also present some robustness checks with small and large stocks.

We employ the same methodology and control variables as documented above, but use small stocks, large stocks, and the size premium (small minus large stocks) as dependent 
variables. Returns of international small and large stocks are proxied for by MSCI size indices for the respective countries. These are available for the time span 1993:1 to 2005:12 and results are shown in $\underline{\text { Table } 6} .^{15}$

It is clear from Panel A (small stocks) and Panel B (large stocks) that earlier U.S. results extend to international markets. There is a significant effect of sentiment on returns for small but not for large stocks. This is very similar to findings in e.g. Lemmon and Portnaguina (2006) or Brown and Cliff (2005) and makes intuitive sense, since small stocks are harder to value and arbitrage than large stocks. The same general picture emerges from Panel C of Table 6 which looks at the size premium. Small stocks are more heavily affected by sentiment shocks than large stocks. Therefore, hypothesis 2 is clearly supported for small stocks.

An obvious objection might be that consumer confidence does not proxy for investor sentiment but rather captures some relevant macro information about time-varying risk premia that is not controlled for by the other macro risk factors already included in our regressions (dividend yield, industrial production, interest rate measures etc.). While our choice of consumer confidence as a sentiment proxy is guided by earlier research showing that consumer confidence contains an irrational element and that it is well suited as a device for tracking noise trader sentiment, one cannot simply rule out a different interpretation completely.

More specifically, if consumer confidence measures time-varying expectations about future economic prospects then one might well observe the negative relation between confidence indices and stock returns, e.g. in an economic setting where higher expected

\footnotetext{
${ }^{15}$ The MSCI indices are not available for the full period from 1985 to 2005 but still cover the major up and down markets oft he late nineties and early 2000s. Furthermore, using MSCI indices facilitates replicability of our results compared to using size indices computed from own calculations which are necessarily based on proprietary data.
} 
economic growth lowers risk aversion of agents and thus lowers expected returns (see e.g. models with habits in consumption such as Campbell and Cochrane (1999)).

However, since consumer confidence contains (a) forward and backward-looking components, and since (b) consumer confidence does not represent expectations of the economy as a whole, an even better predictor should be expected business conditions more generally (see e.g. Campbell and Diebold (2008) who show that expected business conditions negatively forecast stock returns in the U.S.).

In order to test whether consumer confidence contains information about future stock markets over and above that contained in measures of expected business conditions, we re-run our panel regressions with both consumer confidence and expected business conditions (or business sentiment) as predictor variables. If consumer confidence proxies for time-varying expectations about future output movements, then its impact should vanish or be eliminated completely by the inclusion of a genuinely forward-looking proxy for output expectations such as expected business conditions. ${ }^{16}$

Results from this robustness exercise are presented graphically in Figure 1, where we plot the estimated impact of a two standard deviation shock of sentiment (left) and expected business conditions (right) on subsequent returns for forecast horizons of one to 24 months. Results are shown for the aggregate market (Panel A), value (Panel B), and growth stocks (Panel C). As can be seen, consumer confidence remains a statistically and economically significant predictor of returns across our sample countries whereas expected business conditions show no significant forecasting power and also tend to have a positive rather than a negative impact on future returns. Therefore, it seems unlikely that consumer confidence is just a simple business cycle proxy which is not driven to insignificance by the other control

\footnotetext{
${ }^{16}$ We employ the most common business sentiment indicator in each country, e.g. the ISM Index for the U.S., the CBI index for the U.K., or the IFO Index for Germany. Panel unit-root tests indicate that these indices are stationary. More detailed descriptive statistics are available upon request.
} 
variables. ${ }^{17}$ This result also strengthens the empirical support for hypothesis 1 , namely that sentiment has an impact on future returns even after controlling for other fundamental factors.

Finally, we look at the correlation of unexpected returns and sentiment innovations as suggested by Pastor and Stambaugh (2008). The idea in the sentiment-return context here is that in a predictive regression of the form

$$
\begin{gathered}
r_{t+1}^{i}=\delta_{0}^{i}+\delta_{1}^{i} \operatorname{sent}_{t}^{i}+\Upsilon_{t}^{i} \gamma^{i^{\prime}}+\xi_{t+1}^{i} \\
\operatorname{sent}_{t+1}^{i}=\alpha_{0}^{i}+\alpha_{1}^{i} \operatorname{sent}_{t}^{i}+\eta_{t+1}^{i}
\end{gathered}
$$

a plausible result would be that the innovations $\xi_{t}^{i}$, i.e. the unexpected return, and $\eta_{t}^{i}$, i.e. the innovation in noise trader optimism, are positively correlated since it is presumably a wave of unexpected optimism that boosts prices. Therefore, under a behavioral story one would expect to see a positive correlation of $\xi_{t}^{i}$ and $\eta_{t}^{i}$ whereas one would most probably expect to see a negative correlation under a rational story (see the discussion in Pastor and Stambaugh, 2008) where consumer confidence is informative about discount factors.

We report the correlation of $\xi_{t}^{i}$ with $\eta_{t}^{i}$ for all countries $i$ in Table 7. It is obvious that the typical correlation of unexpected returns with sentiment shocks is positive. Furthermore, countries that show a significant relation between returns and sentiment tend to have higher correlation coefficients of the two shocks. This is in line with the story that irrational noise trader sentiment drives price away from fundamentally warranted levels.

\section{Cross-Sectional Analysis}

\subsection{Possible Determinants of Cross-Sectional Variation in the Sentiment-Return Relationship}

\footnotetext{
${ }^{17}$ We also estimate specification (2) on two sub-samples and with a varying number of macro factors included. We do not report the results for brevity but note that our conclusions are qualitatively unchanged.
} 
In this section we discuss possible explanatory variables for the cross-sectional analysis of the sentiment-return relation for our 18 countries. We start by identifying behavioral factors based on the analysis by Chui, Titman and Wei (2008) and then move on to some often used proxies for market efficiency that might drive cross-country results.

\section{Behavioral factors}

The behavioral explanation of the sentiment-return relation says that individuals herd and overreact. Therefore, our findings could be explained by systematic cross-country differences in herd-like overreaction. As noted in the introduction, Chui, Titman and Wei (2008) suggest that differences in collectivistic behavior might be a driver of the tendency of investors to herd. Therefore, we employ a measure of collectivism constructed by Hofstede (2001) which serves to quantify the degree to which people in different countries are programmed to act in groups and not as individuals. ${ }^{18}$

However, herd-like behavior, or correlated behavior across individuals, is not the only ingredient to this behavioral story. Individuals also have to overreact to create the negative relation between sentiment and returns. This point is crucial and is suggested by the findings of Jackson (2004). Jackson shows with broker level trading data for individual investors in Australia, that there is considerable systematic trading by individuals, i.e. trading decision are correlated and do not wash out on an aggregate level. However, he does not find evidence for short-run return reversals after waves of correlated behavior. Therefore, any empirical test of the behavioral story must take into account both dimensions, herding and overreaction.

We employ a second index by Hofstede to capture the likely degree of overreaction across countries. The uncertainty avoidance index (UAI) measures the degree to which a culture programs its members to react to unusual and novel situations. While this is not

\footnotetext{
${ }^{18}$ Chui, Titman and Wei (2005) use the same index to measure individualism which is the original index by Hofstede (2001) where higher values mean higher individualism. We just pre-multiply index values by -1 to obtain our measure for collectivism.
} 
directly addressed in our analysis here, Hofstede documents that people in more uncertainty avoiding countries act and react more emotional compared to countries with low levels of uncertainty avoidance. People in the latter countries act more contemplative and thoughtful. Therefore, we employ the uncertainty avoidance index as a rough proxy for the tendency of individuals to overreact. Furthermore, it is known that UAI is correlated with the collectivism index since the UAI also captures cross-country differences in the tendency of people to follow the same sets of rules and thus behave in the same manner. This is correlated with collectivism and in our sample the correlation between collectivism and uncertainty avoidance indeed is about 0.50 . Therefore, higher levels of the uncertainty avoidance index (UAI) should indicate both a tendency towards more overreaction-like behavior and herd behavior.

\section{Market integrity}

As a second set of explanatory variables we use proxies for what Chui, Titman and Wei (2008) call "stock market integrity". The idea behind these variables is that markets with higher institutional quality should have a more developed flow of information and are consequently more efficient. In order to allow for a direct comparison with Chui, Titman and Wei we include the same variables as in their study.

The market integrity variables include a dummy for the legal origin of a country (the dummy equals one when a country is common law and zero for civil law), the index of antidirector rights, the corruption perception index and accounting standards. These variables are taken from La Porta, Lopez-de-Silanes, Shleifer, and Vishny (1998). Additionally, we follow Chui, Titman and Wei and include the risk of earnings management index. ${ }^{19}$

\footnotetext{
${ }^{19}$ In addition to the market integrity factors, we have also checked other variables such as education, measures of analyst forecast precision, turnover etc. Results on these variables do not add much economic insight and are thus not included to conserve space. They are, however, available upon request.
} 


\subsection{Results}

To investigate the potential determinants of the cross-sectional variation in sentimentreturn relation we pool countries according to high or low values of the above discussed determinants and run panel fixed-effects regressions on the resulting subset of countries. For example, we split the group of all countries into a subset that has collectivism values above the median and a subset with below-median collectivism values. The panel predictive regression in (3) is then estimated separately for these two groups of countries. We can then compare the effect of sentiment on returns to see whether higher collectivism indeed results in stronger sentiment effects. We repeat this procedure for all possible determinants discussed above.

We focus on a twelve months forecast horizon to capture longer-term effects. While results reported below are very similar for other forecasting horizons, we focus on this longer horizon to capture persistent effects of sentiment and not some short-lived effects only.

Results are shown in Table 8 and reveal some interesting results. First, looking at the behavioral factors of Chui, Titman, and Wei, we find that collectivistic countries and countries with a high uncertainty avoidance index show larger effects of sentiment on returns than individualistic and low UAI countries. Indeed, significant effects are obtained for the former groups of countries only and the incremental adj. $\mathrm{R}^{2}$ are significantly higher than for the latter groups of countries.

We furthermore form a composite index of the behavioral factors by principal components analysis which we use to combine the information contained in the two variables. The first principal component explains about $76 \%$ of the total variance. Results for this combined behavioral factor ("PC culture") also show that countries with a cultural tendency for herding and overreaction are subject to a much stronger sentiment-return relation. Therefore, Chui, Titman, and Wei's hypothesis, that collectivism boosts herd-like overreaction (hypothesis 3 in our paper), can be confirmed empirically. 
We next turn to the market integrity factors. ${ }^{20}$ The general picture here is that higher market integrity weakens the sentiment-return relation which seems intuitively reasonable and which is also in line with our third hypothesis. This effect is visible both in the statistical and economic significance of the estimated sentiment coefficients and in the incremental adj. $R^{2} s$. Again we use principal component analysis to combine the integrity factors. ${ }^{21} \mathrm{We}$ show results for the first principal component ("PC market integrity") and find that this general integrity factor significantly discriminates between countries with high and low sentiment effects. Also, the effect of market integrity seems somewhat stronger than that for the behavioral factors.

Finally, we have also run panel regressions on the full set of countries and included (a) interaction terms of sentiment and the culture principal component and/or the market integrity principal component, or (b) dummy variables indicating whether a country has high market integrity and/or high values of the culture principal component. ${ }^{22}$ Results provide the same picture as shown in Table 8 but additionally allow for significance tests of dummy variables or interaction terms which are indicative of whether culture or market integrity significantly affect the sentiment-return relation across countries. The general finding across specifications (i.e. with and without control variables, with one or both principal components included, and for different forecast horizons) is, that differences between the groups of countries shown in Table 8 are significant for both the culture and the market integrity variables. There is, however, no clear pattern that would indicate dominance of one of the two sets of variables, i.e. there is no clear evidence that culture is more important than market integrity or vice versa.

\footnotetext{
${ }^{20}$ In order to make results easier to read in interpret, we have rescaled all market integrity factors such that higher values indicate higher market integrity.

${ }^{21}$ The first principal component explains about $65 \%$ of the total variance.

${ }^{22}$ Results are not included to conserve space but are available upon request.
} 


\section{Conclusions}

We investigate the relation between investor sentiment and future stock returns for 18 industrialized countries and find that sentiment is a significant predictor of expected returns on average across countries. The predictive power of sentiment is most pronounced for short and medium-term horizons of one to six months and washes out over longer horizons of 12 to 24 months. However, the predictive power of sentiment varies across countries and we find in individual country regressions, that sentiment does not contain predictive power for several countries at all.

In order to investigate this issue, we look at possible determinants of the strength of the relation between sentiment and returns and find that the influence of noise traders on markets varies cross-sectionally in a way that is economically intuitive. The impact of sentiment on returns is higher for countries that are culturally more prone to herd-like investment behavior as hypothesized by Chui, Titman and Wei (2008) and for countries that have less efficient regulatory institutions or less market integrity.

Therefore, one cannot simply transfer evidence from the U.S. to other markets and presume that irrational noise traders move stock markets in general. Rather than that, institutional quality and cultural factors are strong determinants of the sentiment-return relation. Regarding possible policy implications, high quality market institutions seem desirable to alleviate effects of noise trading. Culture, however, does not seem to be easily changeable so that sentiment effects should be a persistent phenomenon in countries with a herding culture. 


\section{References}

Amihud, Yakov and Clifford M. Hurvich ,2004. Predictive Regressions: a Reduced-Bias

Estimation Method. Journal of Financial and Quantitative Analysis 39, 813-841.

Ang, Andrew and Geert Bekaert, 2007. Stock Return Predictability: Is it There?. Review of Financial Studies, 20, 651-707.

Ang, Andrew, Robert J. Hodrick, Yuhang Xing, and Xiayoan Zhang, 2008. High

Idiosyncratic Volatility and Low Returns: International and Further U.S. Evidence.

Journal of Financial Economics, forthcoming.

Baker, Malcolm and Jeffrey Wurgler, 2006. Investor Sentiment and the Cross-Section of

Stock Returns. Journal of Finance 61, 1645-1680.

Barber, Brad M, Terrance Odean, and Ning Zhu, 2008. Do Retail Trades Move Markets?.

Review of Financial Studies, forthcoming.

Barber, Brad M, Terrance Odean, and Ning Zhu, 2008a. Systematic Noise. Journal of

Financial Markets, forthcoming.

Black, Fischer, 1986. Noise. Journal of Finance 41, 529-543.

Brown, Gregory W., and Michael T. Cliff, 2004. Investor Sentiment and the Near-Term Stock Market. Journal of Empirical Finance 11, 1-27.

Brown, Gregory W., and Michael T. Cliff, 2005. Investor Sentiment and Asset Valuation. Journal of Business 78, 405-440.

Campbell, John Y. and John H. Cochrane, 1999. By Force of Habit: A Consumption Based Explanation of Aggregate Stock Market Behavior. Journal of Political Economy 107, 205-251.

Campbell, John Y., and Motohiro Yogo, 2006. Efficient Tests of Stock Return Predictability. Journal of Financial Economics 81, 27-60. 
Campbell, Sean D. and Francis X. Diebold, 2008. Stock Return Predictability and Expected Business Conditions: Half a Century of Direct Evidence. Journal of Business and Economic Statistics, forthcoming.

Charoenrook, Anchada, 2003. Does Sentiment Matter?. Working Paper, Vanderbilt University.

Chui, Andy C.W., Sheridan Titman, and K.C. John Wei, 2008. Individualism and Momentum around the World. AFA 2006 Boston Meetings Paper.

Doms, Mark, and Norman Morin, 2004. Consumer Sentiment, the Economy, and the News Media. Working Paper, Federal Reserve Bank of San Francisco.

Fama, Eugene F., and Kenneth R. French, 1998. Value versus Growth: The International Evidence. Journal of Finance 53, 1975-1999.

Fisher, Kenneth L., and Meir Statman, 2003. Consumer Confidence and Stock Returns. Journal of Portfolio Management 30, 115-128.

Gonçalves, Sílvia and Halbert White, 2005. Bootstrap Standard Error Estimates for Linear Regression. Journal of the American Statistical Association 100, 970-979.

Griffin, John M., Xiuqing Ji, and J. Spencer Martin, 2003. Momentum Investing and Business Cycle Risk: Evidence from Pole to Pole. Journal of Finance 58, 2515-2547.

Guiso, Luigi, Paola Sapienza, and Luigi Zingales, 2006. Does Culture Affect Economic Outcomes?. Journal of Economic Perspectives 20, 23-48.

Hodrick, Robert, 1992. Dividend Yields and Expected Stock Returns: Alternative Procedures for Inference and Measurement. Review of Financial Studies 5, 357-386.

Hofstede, Geert, 2001. Culture’s Consequences: Comparing Values, Behaviors, Institutions, and Organizations across Nations. Sage Publication, Beverly Hills.

Hong, Harrison, Walter Torous, and Rossen Valkanov, 2007. Do Industries Lead Stock Markets?. Journal of Financial Economics 83, 367-396. 
Hvidkjaer, Soeren, 2008. Small Trades and the Cross-Section of Stock Returns. Review of Financial Studies 21, 1123-1151.

Jackson, Andrew, 2004. The Aggregate Behaviour of Individual Investors. Working Paper, London Business School.

Jansen, W. Jos, and Niek J. Nahuis, 2003. The Stock Market and Consumer Confidence:

European Evidence. Economics Letters 79, 89-98.

Kaniel, Ron, Gideon Saar, and Sheridan Titman, 2008. Individual Investor Trading and Stock Returns. Journal of Finance, 63 273-310.

Kumar, Alok and Charles Lee, 2006. Retail Investor Sentiment and Return Comovement. Journal of Finance 61, 2451-2486.

La Porta, Rafael, Florencio Lopez-de-Silanes, Andrei Shleifer, and Robert W. Vishny, 1998. Law and Finance. Journal of Political Economy 106, 1113-1155.

Lemmon, Michael, and Evgenia Portniaguina, 2006. Consumer Confidence and Asset Prices: Some Empirical Evidence. Review of Financial Studies 19, 1499-1529.

Levin, Andrew, Lin, Chien-Fu, and James Fu, 2002. Unit Root Tests in Panel Data: Asymptotic and Finite-Sample Properties. Journal of Econometrics 108, 1-24. Lioui, Abraham and Jesper Rangvid, 2007. Stock Return Predictability in a Monetary Economy, Working Paper, University of Copenhagen.

Mark, Nelson C., 1995. Exchange Rates and Fundamentals: Evidence on Long-Horizon Predictability. American Economic Review 85, 201-218.

Menkhoff, Lukas and Rafael Rebitzky, 2008. Investor Sentiment in the US-dollar: Longerterm, Nonlinear Orientation on PPP. Journal of Empirical finance 15, 455-467.

Pastor, Lubos, and Robert Stambaugh, 2008. Predictive Systems: Living with Imperfect Predictors. Journal of Finance, forthcoming.

Qiu, Lily, and Ivo Welch, 2005. Investor Sentiment Measures. Working Paper, Brown University. 
Shiller, Robert J., 2001. Irrational Exuberance. Broadway Books, New York.

Shleifer, Andrei, and Robert Vishny, 1997. The Limits of Arbitrage. Journal of Finance 52, $35-55$.

Schmeling, Maik, 2006. Institutional and Individual Sentiment: Smart Money and Noise Trader Risk?. International Journal of Forecasting 23, 127-145.

Swaminathan, Bhaskaran, 1996. Time-Varying Expected Small Firm Returns and Closed-End Fund Discounts. Review of Financial Studies 9, 845-887.

Valkanov, Rossen, 2003. Long-Horizon Regressions: Theoretical results and applications. Journal of Financial Economics 68, 201-232. 


\section{Appendix 1. Different proxies for investor sentiment}

A natural question that arises when attempting to quantify the influence of sentiment on stock returns is how to measure (unobserved) sentiment. Existing studies have used different proxies, of which closed-end fund discounts are one major vehicle (c.f. Lee, Shleifer and Thaler, 1991, Swaminathan, 1996, or Neal and Wheatley, 1998). Baker and Wurgler (2006) construct a sentiment proxy from several market price based variables such as closedend fund discounts, number of IPO's, turnover etc. Recent studies have started to use micro trading data, such as Kumar and Lee (2006) who use broker data or Barber, Odean and Zhu (2008) who use the TAQ/ISSM data. Finally, some studies use data from investor surveys (cf. Brown and Cliff, 2004 and 2005, or Menkhoff and Rebitzky, 2008). Charoenrook (2003) and Lemmon and Portniaguina (2006) use consumer confidence indexes to proxy for sentiment, based on the observation that Brown and Cliff (2004) find no evidence that closed-end fund discounts reflect sentiment and that Qiu and Welch (2005) report only weak correlation of these fund discounts with UBS/Gallup surveys of investor sentiment. The consumer confidence indexes do better in this respect. Furthermore, Fisher and Statman (2003) provide evidence that consumer confidence correlates well with other sentiment proxies such as the sentiment measure from the American Association of Individual Investors (AAII) whereas Doms and Morin (2004) find that consumer confidence contains an irrational element since it responds to the tone and volume of economics news reports while being hardly affected by the content of news. All these findings make consumer confidence seem to be a reasonable proxy for individual sentiment and we follow these findings by using measures of consumer confidence as a sentiment proxy throughout the paper. 


\section{Appendix 2. Some details on consumer confidence surveys in different countries}

This Appendix provides some details on how consumer confidence surveys are carried out in our different sample countries. The main objective of this appendix is to highlight similarities and possible differences across countries.

There is an internationally standardized set of questions for surveying consumer confidence which is close to the questions asked by the Survey of the University of Michigan. Most surveys in developed countries (and all surveys in our sample countries) make use of these standardized questions to ensure international comparability. More specifically, these questions ask about the past and future financial situation of the household, the past and future economic situation more generally, and about major purchases of durable goods. While these questions are core, most surveys ask additional questions which are often similar across countries. For example, various surveys (e.g. the surveys of the European Commission and the polls in Switzerland) ask for the expected employment situation over the next year and CPI developments, and some surveys additionally ask for longer-term expectations (e.g. 5 year forecasts in Australia). ${ }^{23}$ However, given that core questions are extremely similar across developed countries, one might expect that consumer confidence indices are comparable internationally.

While there do not seem to be economically interesting differences between survey questions, maybe the clearest difference between countries occurs with respect to seasonal adjustments. Although most countries do seasonally adjust (Switzerland is an exception), they do not use the same procedures. For example, Japan uses "X-11" whereas the European commission employs "Dainties". Whether employment of these different procedures may affect econometric estimates quantitatively, there is little evidence in our results that it matters qualitatively. Significant impacts of consumer confidence on returns can be found in countries

\footnotetext{
${ }^{23}$ There are also less common questions. The Japanese survey for example includes a question about planned travels and holidays which does not seem to be included elsewhere.
} 
like Switzerland (no seasonal adjustments), Japan (X-11), and Germany (Dainties), but not in e.g. Ireland (which is also adjusted by Dainties).

Another difference between surveys worth mentioning are the questions about future developments. While most surveys (e.g. the European surveys) mainly ask for one year horizons, there are exceptions such as the Michigan survey which asks for one year horizons regarding a household's financial situation but for 5 year horizons regarding economic developments. Therefore, forecast horizons differ somewhat between countries.

Finally, consumer confidence surveys in different countries naturally differ by the number of participants. While most surveys are based on more than 1,000 households, there are large differences between smaller countries (e.g. Switzerland with 1,100 participants) and large countries (e.g. France with 3,300 participants, Japan with more than 5,000 participants, and obvious outliers such as the Michigan survey in the U.S. which is based on 500 respondents, only. 


\section{Appendix 3. Details on the bias adjustments}

Standard econometric inference in regressions of the form in (2), most probably yields biased estimates of the slope coefficients. Several authors (see Stambaugh, 1999, Valkanov, 2003, or Ferson et al., 2003) have documented this problem, which is caused by highly persistent regressors. In this case OLS estimation results are still consistent but suffer more than likely from severe biases in finite samples. For simple regressions with only one predictor it can be shown analytically that the bias in coefficient point estimates increases in the degree of persistence of the regressor (see Stambaugh, 1999). As shown in Table 1, consumer confidence indexes are highly persistent. ${ }^{24} \mathrm{~A}$ further complication arises from the overlapping the dependent variable, which induces a moving average structure of order $(k-1)$ to the error terms.

Several authors (e.g. Brown and Cliff, 2005) rely on some form of simulation procedure to account for biased coefficient estimates and standard errors. Another way to quantify and to adjust for biases is to use auxiliary regressions (Amihud and Hurvich, 2004). ${ }^{25}$ Some authors have recently switched to using Hodrick (1992) standard errors (e.g. Ang and Bekaert, 2007, or Lioui and Rangvid, 2007) and do not bias-adjust coefficient estimates.

We use a moving block-bootstrap as suggested by Gonçalves and White (2005) to account for biased coefficient estimates and standard errors. The exact procedure is as follows. We first estimate the predictive regression (panel regression or the system of predictive regressions with different forecasting horizons as detailed in the main text). Coefficients are stored. We then repeatedly bootstrap the raw data in blocks with a block length of 10 observations to generate 10,000 new time series generated under the null of no predictability for all dependent and explanatory variables. The block length is chose to be

\footnotetext{
${ }^{24}$ Brown and Cliff (2005) also find individual sentiment from direct investor surveys in the U.S. to be highly correlated over time. Therefore, the high degree of persistence is not special to the consumer confidence indices employed here.

${ }^{25}$ Campbell and Yogo (2006) provide a method for efficient tests of stock return predictability in the presence of near unit-root regressors. However, their method does not extend directly to multiple regressors and multi-period forecasts.
} 
large since confidence indices are very persistent. Choosing smaller or longer block lengths does not seem to affect our results in an interesting way. Predictive regressions are then estimated on these 10,000 artificial time series to obtain the bootstrap distribution of coefficient estimates.

We report bias-adjusted coefficient estimates, i.e. we subtract the mean of the bootstrap coefficient estimates from the original bootstrap estimate. P-values reported in the tables are based on the share of simulated t-statistics exceeding the estimated t-statistic from the original regression.

The results for individual countries in Table 5 are based on systems of equations

$$
\begin{gathered}
\mathrm{r}_{\mathrm{t}+1}^{\mathrm{i}}=\delta_{0}^{\mathrm{i},(1)}+\delta_{1}^{\mathrm{i},(1)} \operatorname{sent}_{\mathrm{t}}^{\mathrm{i}}+\Psi_{\mathrm{t}}^{\mathrm{i},(1)} \gamma^{\mathrm{i},(1)}+\xi_{\mathrm{t}+1}^{\mathrm{i},(1)} \\
\frac{1}{\kappa} \sum_{\kappa=1}^{6} \mathrm{r}_{\mathrm{t}+\kappa}^{\mathrm{i}}=\delta_{0}^{\mathrm{i},(6)}+\delta_{1}^{\mathrm{i},(6)} \operatorname{sent}_{\mathrm{t}}^{\mathrm{i}}+\Psi_{\mathrm{t}}^{\mathrm{i},(6)} \gamma^{\mathrm{i},(6)}+\xi_{\mathrm{t}+1 \mapsto \mathrm{t}+6}^{\mathrm{i},(6)} \\
\frac{1}{\kappa} \sum_{\kappa=1}^{12} \mathrm{r}_{\mathrm{t}+\kappa}^{\mathrm{i}}=\delta_{0}^{\mathrm{i},(12)}+\delta_{1}^{\mathrm{i},(12)} \operatorname{sent}_{\mathrm{t}}^{\mathrm{i}}+\Psi_{\mathrm{t}}^{\mathrm{i},(12)} \gamma^{\mathrm{i},(12)}+\xi_{\mathrm{t}+1 \mapsto \mathrm{t}+12}^{\mathrm{i}(12)} \\
\frac{1}{\kappa} \sum_{\kappa=1}^{24} \mathrm{r}_{\mathrm{t}+\kappa}^{\mathrm{i}}=\delta_{0}^{\mathrm{i},(24)}+\delta_{1}^{\mathrm{i},(24)} \operatorname{sent}_{\mathrm{t}}^{\mathrm{i}}+\Psi_{\mathrm{t}}^{\mathrm{i},(24)} \gamma^{\mathrm{i},(24)}+\xi_{\mathrm{t}+1 \mapsto \mathrm{t}+24}^{\mathrm{i},(24)}
\end{gathered}
$$

for each country $i$. This system is estimated via GMM (exactly identified) and significance tests are carried out via the same bootstrap procedure as employed above. 


\section{Table 1. Descriptive Statistics}

This table shows descriptive statistics for all countries used in the analysis. In particular, the table shows the start month of the sample (all series end in December 2005) and the source of the data. Furthermore, it shows means $(\mu)$ and standard deviations $(\sigma)$ for the market return, returns of value stocks, and growth stocks. Finally, the last three columns show the mean, standard deviation and first order autocorrelation for consumer confidence indices.

\begin{tabular}{|c|c|c|c|c|c|c|c|c|c|c|c|c|}
\hline \multirow{2}{*}{ Country } & \multirow{2}{*}{ Label } & \multirow{2}{*}{ Start } & \multirow{2}{*}{ Source } & \multicolumn{2}{|c|}{ Market } & \multicolumn{2}{|c|}{ Value } & \multicolumn{2}{|c|}{ Growth } & \multicolumn{3}{|c|}{ Consumer Confidence } \\
\hline & & & & $\mu$ & $\sigma$ & $\mu$ & $\sigma$ & M & $\sigma$ & $\mu$ & $\sigma$ & $\rho(1)$ \\
\hline Australia & ATRL & $1985 \mathrm{M} 1$ & Datastream & 1.24 & 4.86 & 1.55 & 5.11 & 1.06 & 5.55 & 100.59 & 12.66 & 0.92 \\
\hline Austria & ATR & $1996 \mathrm{M}$ & DG ECFIN & 1.40 & 4.67 & 1.91 & 6.45 & 0.80 & 4.54 & -1.36 & 6.41 & 0.91 \\
\hline Belgium & BEL & 1985 M1 & DG ECFIN & 1.29 & 5.09 & 1.83 & 6.69 & 1.13 & 5.30 & -7.00 & 9.53 & 0.95 \\
\hline Denmark & DEN & 1989 M1 & DG ECFIN & 1.06 & 5.13 & 1.24 & 5.93 & 1.03 & 6.10 & 5.38 & 8.36 & 0.95 \\
\hline Finland & FIN & 1995 M11 & DG ECFIN & 1.46 & 8.97 & 1.54 & 7.16 & 1.69 & 10.90 & 14.90 & 3.84 & 0.89 \\
\hline France & FRA & 1985 M1 & DG ECFIN & 1.23 & 5.89 & 1.54 & 6.99 & 1.10 & 5.83 & -18.60 & 8.49 & 0.94 \\
\hline Germany & GER & 1986 M1 & DG ECFIN & 0.79 & 6.16 & 1.42 & 6.65 & 0.69 & 6.93 & -8.98 & 8.79 & 0.97 \\
\hline Ireland & IRE & $1991 \mathrm{M} 1$ & DG ECFIN & 1.33 & 5.26 & 1.87 & 7.66 & 1.05 & 6.33 & -3.87 & 13.52 & 0.97 \\
\hline Italy & ITA & 1985 M1 & DG ECFIN & 1.29 & 7.09 & 1.25 & 8.14 & 1.26 & 7.20 & -12.78 & 7.06 & 0.93 \\
\hline Japan & JAP & 1985 M1 & Datastream & 0.49 & 5.80 & 1.11 & 6.70 & 0.20 & 6.40 & 43.26 & 4.62 & 0.97 \\
\hline Netherlands & NET & $1985 \mathrm{M} 1$ & DG ECFIN & 1.11 & 5.07 & 1.62 & 7.15 & 1.04 & 4.90 & 4.02 & 11.68 & 0.97 \\
\hline New Zealand & NEWZ & 1989 M1 & Datastream & 0.64 & 5.30 & -0.35 & 8.51 & 0.80 & 5.95 & 112.95 & 12.00 & 0.99 \\
\hline Norway & NOR & 1992 M9 & EcoWin & 1.44 & 5.86 & 2.05 & 9.57 & 1.27 & 5.97 & 20.06 & 13.38 & 0.97 \\
\hline Spain & SPA & $1988 \mathrm{M} 1$ & DG ECFIN & 1.20 & 5.75 & 1.74 & 5.69 & 0.77 & 6.28 & -10.34 & 8.96 & 0.95 \\
\hline Sweden & SWE & 1995 M9 & DG ECFIN & 1.29 & 6.69 & 1.65 & 6.53 & 1.10 & 8.35 & 7.39 & 7.21 & 0.94 \\
\hline Switzerland & SWI & 1985 M1 & Datastream & 1.08 & 4.97 & 1.31 & 6.82 & 0.97 & 4.84 & -10.83 & 21.66 & 0.99 \\
\hline United Kingdom & UK & 1985 M1 & DG ECFIN & 1.07 & 4.64 & 1.25 & 5.48 & 0.99 & 4.75 & -8.25 & 7.81 & 0.93 \\
\hline United States & US & 1985 M1 & Datastream & 1.08 & 4.43 & 1.23 & 4.10 & 1.09 & 4.88 & 95.29 & 12.90 & 0.84 \\
\hline
\end{tabular}




\section{Table 2. Panel unit-root tests}

This Table shows panel unit-root tests for consumer confidence indices. The test by Levin, Lin, and Chu tests the null of a unit root assuming a common unit root process. The other two procedures test the null of a unit root assuming individual unit root processes. The lag length selection is based on SIC and the test equation contains individual intercepts. Stars refer to the level of significance: ***: $0.01, * *: 0.05, *: 0.10$.

\begin{tabular}{lccc}
\hline & test statistic & p-value & obs \\
\hline Levin, Lin, and Chu & -2.88 & $* * *(0.00)$ & 3,795 \\
Im, Pesaran, and Shin & -5.80 & $* * *(0.00)$ & 3,795 \\
PP-Fisher & 90.50 & $* * *(0.00)$ & 3,819 \\
\hline
\end{tabular}

\section{Table 3. Granger-causality tests}

Reported are pairwise Granger-causality tests for sentiment and returns and tests for blockexogeneity. The latter are obtained from VAR models which include returns, sentiment, and predetermined control variables $(\Psi)$. The lag length is chosen by minimizing SIC. Stars refer to the level of significance: ***: $0.01, * *: 0.05, *: 0.10$.

\begin{tabular}{lccc}
\hline & Aggregate market & Value stocks & Growth stocks \\
\hline Pairwise & $* * *(0.00)$ & $* * *(0.00)$ & $* * *(0.00)$ \\
$\mathrm{r} \rightarrow$ sent & $* *(0.02)$ & $* * *(0.00)$ & $*(0.06)$ \\
$\mathrm{sent} \rightarrow \mathrm{r}$ & & & \\
\hline Block Exogeneity & $* * *(0.00)$ & $* * *(0.00)$ & $* * *(0.00)$ \\
$\mathrm{r} \rightarrow$ sent & $* *(0.01)$ & $* * *(0.00)$ & $* *(0.05)$ \\
sent $\rightarrow \mathrm{r}$ & & &
\end{tabular}




\section{Table 4. Panel fixed effects regressions}

This table shows results for panel fixed effects regressions with future stock returns as dependent variable and sentiment (sent) as well as several control variables (not shown in the tables) as predictive variables. $\triangle$ adj. $\mathrm{R}^{2}$ denotes the change in adj. $\mathrm{R}^{2}$ when sentiment is included as an additional regressor in the equation. Stars refer to the level of significance: $* * *: 0.01, * *: 0.05, *: 0.10$.

Panel A: Aggregate market returns

\begin{tabular}{lrrrr}
\hline & \multicolumn{4}{c}{ Forecast horizon } \\
\cline { 2 - 5 } & 1 month & 6 months & 12 months & 24 months \\
\hline Sent & -0.42 & -0.31 & -0.26 & -0.20 \\
p-value & $* *(0.01)$ & $* *(0.04)$ & $* *(0.05)$ & $*(0.06)$ \\
\hline adj. $\mathbf{R}^{2}$ & 0.01 & 0.06 & 0.11 & 0.19 \\
$\triangle$ adj. $\mathrm{R}^{2}$ & 0.01 & 0.02 & 0.03 & 0.04 \\
Obs & 3,702 & 3,612 & 3,504 & 3,288 \\
\hline
\end{tabular}

Panel B: Returns of value stocks

\begin{tabular}{lrrrr}
\hline & \multicolumn{4}{c}{ Forecast horizon } \\
\cline { 2 - 5 } & 1 month & 6 months & 12 months & 24 months \\
\hline Sent & -0.70 & -0.55 & -0.45 & -0.28 \\
p-value & $* * *(0.00)$ & $* * *(0.00)$ & $* *(0.01)$ & $* *(0.02)$ \\
\hline adj. $\mathrm{R}^{2}$ & 0.01 & 0.09 & 0.14 & 0.20 \\
$\triangle$ adj. $\mathrm{R}^{2}$ & 0.01 & 0.04 & 0.04 & 0.04 \\
Obs & 3,702 & 3,612 & 3,504 & 3,288 \\
\hline
\end{tabular}

Panel C: Returns of growth stocks

\begin{tabular}{lrrrr}
\hline & \multicolumn{4}{c}{ Forecast horizon } \\
\cline { 2 - 5 } & 1 month & 6 months & 12 months & 24 months \\
\hline Sent & -0.40 & -0.30 & -0.27 & -0.21 \\
p-value & $* * *(0.00)$ & $* *(0.05)$ & $*(0.06)$ & $*(0.05)$ \\
\hline adj. $\mathrm{R}^{2}$ & 0.01 & 0.06 & 0.11 & 0.22 \\
$\triangle$ adj. $\mathrm{R}^{2}$ & 0.01 & 0.02 & 0.02 & 0.04 \\
Obs & 3,702 & 3,612 & 3,504 & 3,288 \\
\hline
\end{tabular}




\section{Table 5. Return predictability across horizons}

This table shows results for predictive regressions with future stock returns as dependent variable and sentiment (sent) as well as several control variables (not shown in the tables) as predictive variables. Shown are mean coefficients across forecast horizons of 1, 6, 12, and 24 months. The p-value refers to the test that coefficients across forecast horizons are jointly equal to zero. Stars refer to the level of significance: ***: $0.01, * *: 0.05, *: 0.10$.

Panel A: Aggregate Market

\begin{tabular}{lrrlrr}
\hline & mean coef & p-val. & mean coef & p-val. \\
\hline ATR & -0.36 & $*(0.07)$ & JAP & -0.73 & $* * *(0.00)$ \\
ATRL & 0.01 & $(0.76)$ & NET & -0.09 & $(0.44)$ \\
BEL & -0.29 & $* * *(0.00)$ & NEWZ & 0.19 & $(0.50)$ \\
DEN & 0.06 & $(0.91)$ & NOR & -0.54 & $* *(0.05)$ \\
FIN & 0.40 & $(0.69)$ & SPA & -0.25 & $*(0.09)$ \\
FRA & -0.54 & $* *(0.02)$ & SWE & -0.65 & $* *(0.03)$ \\
GER & -0.65 & $* * *(0.01)$ & SWI & -0.69 & $* *(0.02)$ \\
IRE & -0.04 & $(0.81)$ & UK & -0.10 & $(0.21)$ \\
ITA & -0.57 & $* * *(0.00)$ & US & -0.21 & $* *(0.04)$ \\
\hline
\end{tabular}

Panel B: Value stocks

\begin{tabular}{lrrlrr}
\hline & mean coef & p-val. & mean coef & p-val. \\
\hline ATR & -0.44 & $* *(0.03)$ & JAP & -0.71 & $* * *(0.00)$ \\
ATRL & -0.07 & $(0.32)$ & NET & -0.11 & $*(0.09)$ \\
BEL & -0.54 & $* *(0.01)$ & NEWZ & 0.32 & $*(0.09)$ \\
DEN & 0.14 & $(0.29)$ & NOR & -0.69 & $* * *(0.00)$ \\
FIN & -0.44 & $* *(0.04)$ & SPA & -0.02 & $(0.71)$ \\
FRA & -0.47 & $*(0.06)$ & SWE & -0.91 & $* * *(0.01)$ \\
GER & -0.51 & $* *(0.04)$ & SWI & -0.70 & $* *(0.02)$ \\
IRE & 0.04 & $(0.87)$ & UK & -0.21 & $*(0.06)$ \\
ITA & -0.82 & $* * *(0.00)$ & US & -0.25 & $* *(0.05)$ \\
\hline
\end{tabular}

Panel C: Growth stocks

\begin{tabular}{lrrlrr}
\hline & mean coef & p-val. & mean coef & p-val. \\
\hline ATR & -0.21 & $*(0.07)$ & JAP & -0.24 & $* *(0.05)$ \\
ATRL & -0.02 & $(0.85)$ & NET & -0.13 & $*(0.07)$ \\
BEL & -0.70 & $* *(0.01)$ & NEWZ & 0.18 & $(0.34)$ \\
DEN & -0.19 & $(0.11)$ & NOR & -0.21 & $* *(0.04)$ \\
FIN & 0.29 & $(0.41)$ & SPA & -0.40 & $*(0.08)$ \\
FRA & -0.37 & $* *(0.05)$ & SWE & -0.52 & $* *(0.05)$ \\
GER & -0.52 & $* *(0.04)$ & SWI & -0.68 & $* * *(0.00)$ \\
IRE & -0.08 & $(0.66)$ & UK & -0.11 & $(0.10)$ \\
ITA & -0.77 & $* * *(0.01)$ & US & -0.11 & $(0.11)$ \\
\hline
\end{tabular}




\section{Table 6. Small versus large stocks}

This table replicates Table 4 but shows results for small and large stocks (i.e. stocks sorted on market capitalization). Panel $\mathrm{C}$ shows results for the long-short portfolio which is long in large cap stocks and short in small cap stocks. The data used for large and small stocks is based on MSCI size indices. The sample is shorter due to data availability and ranges from 1993M1 to 2005M12. $\triangle$ adj. $\mathrm{R}^{2}$ denotes the change in adj. $\mathrm{R}^{2}$ when sentiment is included as an additional regressor in the equation. Stars refer to the level of significance: ***: $0.01, * *$ : $0.05, *: 0.10$.

Panel A: Large stocks

\begin{tabular}{lrrrr}
\hline & \multicolumn{4}{c}{ Forecast horizon } \\
\cline { 2 - 5 } & 1 month & 6 months & 12 months & 24 months \\
\hline Sent & -0.27 & -0.32 & -0.21 & -0.19 \\
p-value & $(0.19)$ & $(0.10)$ & $(0.26)$ & $(0.44)$ \\
\hline adj. $\mathbf{R}^{2}$ & 0.01 & 0.05 & 0.11 & 0.19 \\
$\triangle$ adj. $\mathrm{R}^{2}$ & 0.00 & 0.01 & 0.01 & 0.01 \\
Obs & 2,391 & 2,296 & 2,182 & 1,965 \\
\hline
\end{tabular}

Panel B: Small stocks

\begin{tabular}{lrrrr}
\hline & \multicolumn{4}{c}{ Forecast horizon } \\
\cline { 2 - 5 } & 1 month & 6 months & 12 months & 24 months \\
\hline Sent & -0.74 & -0.61 & -0.44 & -0.24 \\
p-value & $* * *(0.00)$ & $* * *(0.00)$ & $* * *(0.00)$ & $* *(0.02)$ \\
\hline adj. $\mathrm{R}^{2}$ & 0.02 & 0.12 & 0.20 & 0.22 \\
$\triangle$ adj. $\mathrm{R}^{2}$ & 0.01 & 0.03 & 0.04 & 0.04 \\
Obs & 2,391 & 2,296 & 2,182 & 1,965 \\
\hline
\end{tabular}

Panel C: Size premium

\begin{tabular}{lrrrr}
\hline & \multicolumn{4}{c}{ Forecast horizon } \\
\cline { 2 - 5 } & 1 month & 6 months & 12 months & 24 months \\
\hline Sent & -0.33 & -0.28 & -0.16 & -0.04 \\
p-value & $* *(0.02)$ & $* *(0.05)$ & $(0.11)$ & $(0.84)$ \\
\hline adj. $\mathbf{R}^{2}$ & 0.01 & 0.05 & 0.07 & 0.09 \\
$\triangle$ adj. $\mathrm{R}^{2}$ & 0.01 & 0.01 & 0.00 & 0.00 \\
Obs & 2,391 & 2,296 & 2,182 & 1,965 \\
\hline
\end{tabular}




\section{Figure 1. Consumer Sentiment and Business Sentiment}

This figure shows estimated coefficients and 95\% confidence intervals for regressions of average k-period stock returns on consumer sentiment (left side) and business sentiment (right side). The $\mathrm{x}$-axis measures the forecast horizon $(1,2, \ldots, 24$ months) and the y-axis shows the impact of a two standard deviation sentiment shock on subsequent returns.

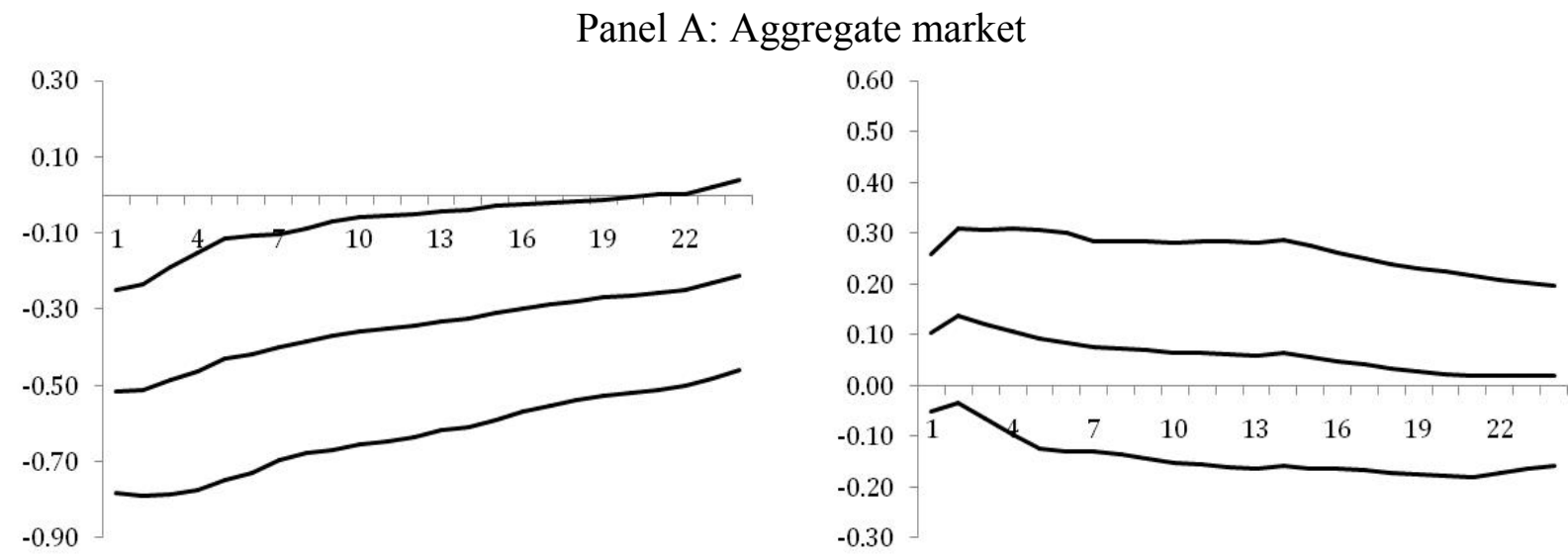

Panel B: Value stocks
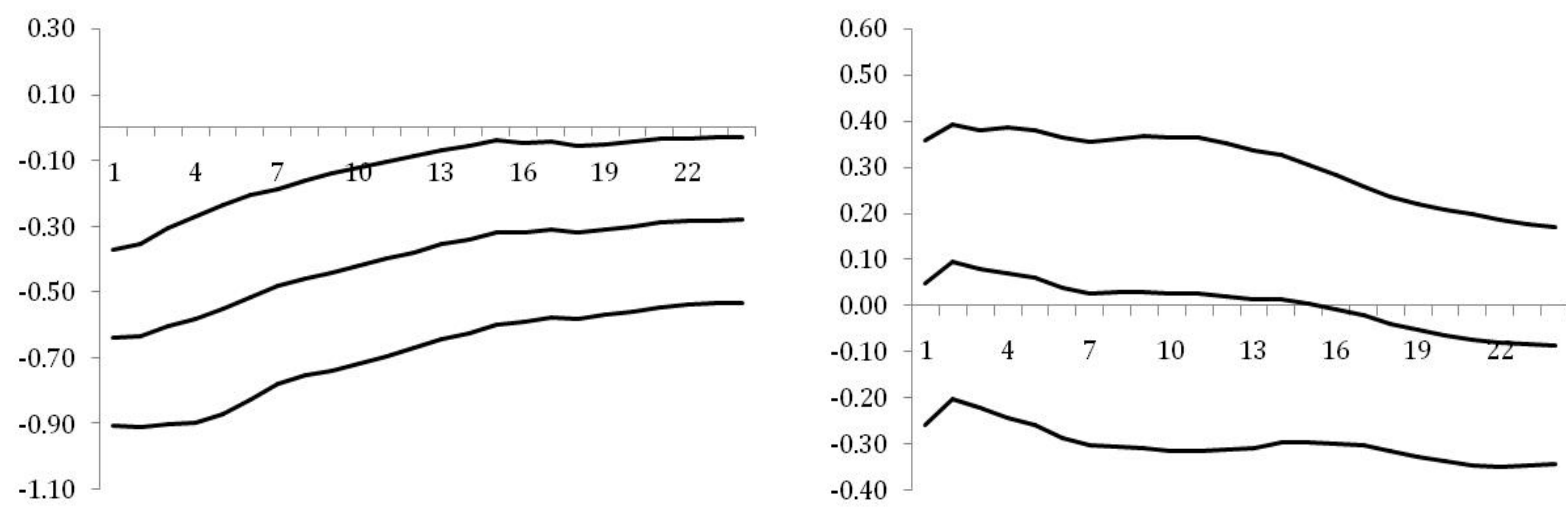

Panel C: Growth stocks
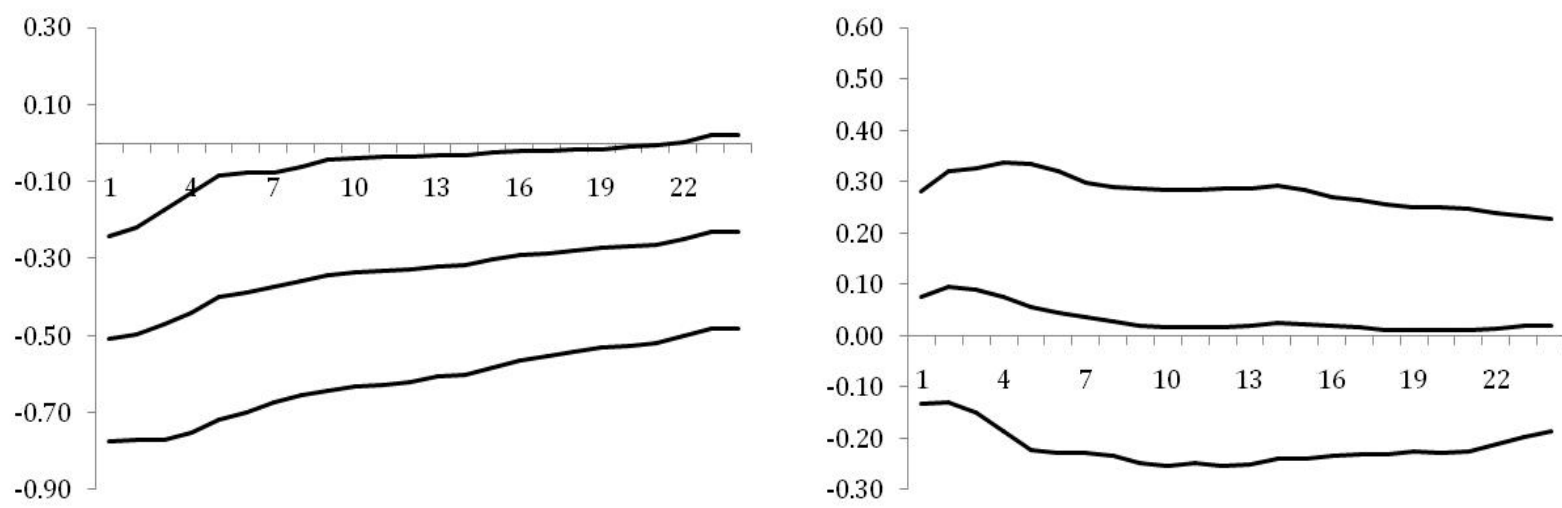
Table 7. Correlation of consumer confidence innovations and unexpected returns

This table shows correlation coefficients for unexpected returns and sentiment innovations from the predictive system in equations (4) and (5) for market returns and returns of value and growth stocks. Stars refer to the level of significance: ***: $0.01, * *: 0.05, *: 0.10$.

\begin{tabular}{|c|c|c|c|}
\hline & $\begin{array}{l}\text { Aggregate } \\
\text { Market }\end{array}$ & $\begin{array}{l}\text { Value } \\
\text { stocks }\end{array}$ & $\begin{array}{c}\text { Growth } \\
\text { stocks }\end{array}$ \\
\hline ATRL & 0.03 & 0.05 & 0.04 \\
\hline ATR & 0.13 & 0.03 & 0.11 \\
\hline BEL & 0.08 & 0.02 & 0.12 \\
\hline DEN & 0.02 & 0.06 & 0.02 \\
\hline FIN & 0.03 & -0.03 & 0.04 \\
\hline FRA & 0.14 & 0.16 & 0.12 \\
\hline GER & 0.02 & 0.02 & 0.01 \\
\hline IRE & 0.03 & 0.09 & 0.07 \\
\hline ITA & 0.09 & 0.10 & 0.07 \\
\hline JAP & 0.10 & 0.16 & 0.07 \\
\hline NET & 0.13 & 0.14 & 0.12 \\
\hline NEWZ & 0.20 & -0.02 & 0.22 \\
\hline NOR & 0.15 & 0.10 & 0.13 \\
\hline SPA & 0.16 & 0.07 & 0.17 \\
\hline SWE & 0.15 & 0.15 & 0.11 \\
\hline SWI & 0.02 & 0.05 & 0.02 \\
\hline UK & 0.12 & 0.12 & 0.12 \\
\hline US & 0.12 & 0.17 & 0.10 \\
\hline Average & $\begin{array}{c}0.10 \\
* * *(0.00)\end{array}$ & $\begin{array}{c}0.08 \\
* * *(0.00)\end{array}$ & $\begin{array}{c}0.09 \\
* * *(0.00)\end{array}$ \\
\hline
\end{tabular}




\section{Table 8. Cross-sectional analysis of the sentiment-return relation}

This table shows results for panel fixed effects regressions where countries are pooled according to one of the determinants shown in the first column. Specifically, countries are allocated to one of two groups depending on whether they are above or below the median of a specific determinant. $\triangle$ adj. $\mathrm{R}^{2}$ denotes the change in adj. $\mathrm{R}^{2}$ when sentiment is included as an additional regressor in the equation. Stars refer to the level of significance: ***: 0.01, **: $0.05, *: 0.10$.

\begin{tabular}{|c|c|c|c|c|}
\hline & \multicolumn{2}{|c|}{ Countries above median } & \multicolumn{2}{|c|}{ Countries below median } \\
\hline & coef. & $\triangle \operatorname{adj} . R^{2}$ & coef. & $\triangle \operatorname{adj} . R^{2}$ \\
\hline \multicolumn{5}{|l|}{ Behavioral factors } \\
\hline Collectivism & $\begin{array}{r}-0.34 \\
*(0.09)\end{array}$ & 0.05 & $\begin{array}{l}-0.19 \\
(0.29)\end{array}$ & 0.01 \\
\hline Uncertainty avoidance & $\begin{array}{r}-0.51 \\
* *(0.02)\end{array}$ & 0.06 & $\begin{array}{r}0.01 \\
(0.91)\end{array}$ & 0.00 \\
\hline PC culture & $\begin{array}{r}-0.39 \\
* *(0.04) \\
\end{array}$ & 0.05 & $\begin{array}{r}-0.07 \\
(0.42) \\
\end{array}$ & 0.00 \\
\hline \multicolumn{5}{|l|}{ Market integrity } \\
\hline Anti-director rights & $\begin{array}{r}0.02 \\
(0.81)\end{array}$ & 0.00 & $\begin{array}{r}-0.59 \\
* *(0.03)\end{array}$ & 0.10 \\
\hline Corruption perception & $\begin{array}{l}-0.11 \\
(0.52)\end{array}$ & 0.01 & $\begin{array}{r}-0.40 \\
*(0.06)\end{array}$ & 0.04 \\
\hline Accounting standards & $\begin{array}{r}-0.04 \\
(0.69)\end{array}$ & 0.00 & $\begin{array}{r}-0.35 \\
(0.17)\end{array}$ & 0.03 \\
\hline Earnings management & $\begin{array}{l}-0.03 \\
(0.53)\end{array}$ & 0.01 & $\begin{array}{r}-0.55 \\
* *(0.02)\end{array}$ & 0.08 \\
\hline PC market integrity & $\begin{array}{r}-0.03 \\
(0.71)\end{array}$ & 0.00 & $\begin{array}{r}-0.54 \\
* *(0.05)\end{array}$ & 0.08 \\
\hline
\end{tabular}

